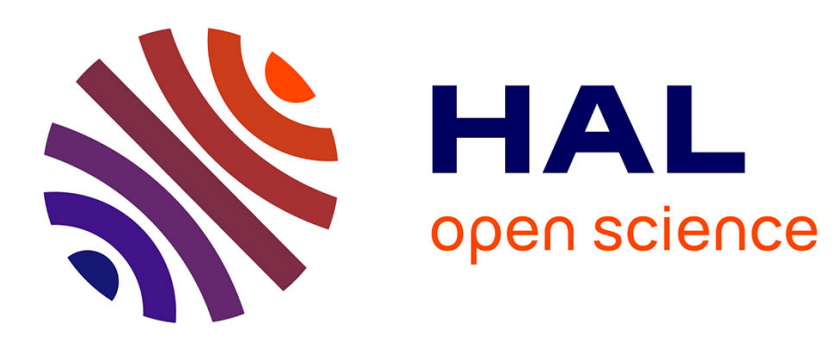

\title{
Löwner-Based Tensor Decomposition for Blind Source Separation in Atrial Fibrillation ECGs
}

\author{
Pedro Marinho R. de Oliveira, Vicente Zarzoso
}

\section{To cite this version:}

Pedro Marinho R. de Oliveira, Vicente Zarzoso. Löwner-Based Tensor Decomposition for Blind Source Separation in Atrial Fibrillation ECGs. EUSIPCO-2019, 27th European Signal Processing Conference, Sep 2019, A Coruña, Spain. hal-02271029

\section{HAL Id: hal-02271029 https://hal.science/hal-02271029}

Submitted on 26 Aug 2019

HAL is a multi-disciplinary open access archive for the deposit and dissemination of scientific research documents, whether they are published or not. The documents may come from teaching and research institutions in France or abroad, or from public or private research centers.
L'archive ouverte pluridisciplinaire HAL, est destinée au dépôt et à la diffusion de documents scientifiques de niveau recherche, publiés ou non, émanant des établissements d'enseignement et de recherche français ou étrangers, des laboratoires publics ou privés. 


\title{
Löwner-Based Tensor Decomposition for Blind Source Separation in Atrial Fibrillation ECGs
}

\author{
Pedro Marinho R. de Oliveira and Vicente Zarzoso \\ Université Côte d'Azur, CNRS, I3S Laboratory \\ CS 40121, 06903 Sophia Antipolis Cedex, France \\ Email: \{marinho, zarzoso $\} @ i 3 s . u n i c e . f r$
}

\begin{abstract}
The estimation of the atrial activity (AA) signal in electrocardiogram (ECG) recordings is an important step in the noninvasive analysis of atrial fibrillation (AF), the most common sustained cardiac arrhythmia in clinical practice. Recently, this blind source separation (BSS) problem has been formulated as a tensor factorization, based on the block term decomposition (BTD) of a data tensor built from Hankel matrices of the observed ECG. However, this tensor factorization technique was precisely assessed only in segments with long $R-R$ intervals and with the AA well defined in the TQ segment, where ventricular activity (VA) is absent. Due to the chaotic nature of AA in AF, segments with disorganized or weak $A A$ and with short $R-R$ intervals are quite more common in persistent $\mathrm{AF}$, posing some difficulties to the BSS methods to extract the AA signal, regarding performance and computational cost. In this paper, the BTD built from Löwner matrices is proposed as a method to separate VA from $\mathrm{AA}$ in these challenging scenarios. Experimental results obtained in a population of 10 patients show that the Löwner-based BTD outperforms the Hankel-based BTD and two well-known matrixbased methods in terms of atrial signal estimation quality and computational cost.
\end{abstract}

Index Terms-Block Term Decomposition, Blind Source Separation, Löwner Matrices, Atrial Fibrillation, Electrocardiogram

\section{INTRODUCTION}

Decreasing life quality and increasing healthcare costs, atrial fibrillation (AF) is a supraventricular tachyarrhythmia characterized by an uncordinated and irregular atrial activation [1]. Persistent AF represents a particularly complex case of this arrhythmia, where extensive atrial remodelling has taken place due to sustained AF, significantly affecting atrial activity (AA) and AF perpetuation itself. This challenging cardiac condition represents an economical burden, as a patient with AF spends, anually, approximately $\$ 8700$ more in healthcare than a patient without AF. Also a major health and social concern, AF has about 467000 hospitalizations as the primary diagnosis every year in USA, and the number of deaths are estimated to be more than 99000 [1]. With similar numbers present in Europe, this heart rhythm disorder can become a new epidemic by 2050 [2]. Considering this scenario and the fact that the mechanisms of $\mathrm{AF}$ are complex and not completely understood, intensive clinical research into this

Pedro Marinho R. de Oliveira is funded by a PhD scholarship from the IT Doctoral School (EDSTIC) of the Université Côte d'Azur. cardiac rhythm disturbance has increased in the past few years and is expected to increase further.

In the electrocardiogram (ECG), the $\mathrm{P}$ wave corresponds to a normal atrial depolarization during normal sinus rhythm. AF is characterized by irregular R-R intervals and the replacement of the $\mathrm{P}$ wave by $\mathrm{f}$ waves (fibrillatory waves), which are present throughout the whole ECG recording. However, they are masked by the QRS-T complex of ventricular depolarization and repolarization, i.e., the ventricular activity (VA), in each heartbeat.

Signal processing techniques are important and necessary tools to noninvasively separate the AA from the standard 12lead ECG for a precise analysis and characterization of the $f$ waves, in order to better understand the complex mechanisms behind AF. The extraction of AA from multi-lead ECGs accepts a blind source separation (BSS) formulation [3]. Methods based on matrix decompositions to solve BSS problems, such as principal component analysis (PCA) [4] and independent component analysis (ICA) [5] are reported in the literature as useful tools in noninvasive AA extraction [3], [6]. Nonetheless, the uniqueness of matrix decompositions are just guaranteed up to some constraints and, although mathematically convenient, such constraints may lack physiological grounds, making difficult the results interpretation.

Overcoming this limitation of matrix decompositions, tensor decompositions present some remarkable features over matrixbased techniques, for example, their essential uniqueness with minimal or no constraints. The block term decomposition (BTD) built from Hankel matrices was recently proposed to noninvasively extract the AA signal from AF ECGs [7]. This tensor factorization technique has been evaluated in experiments using synthetic and real data, showing that it can outperform the matrix-based techniques for noninvasive AA extraction in short and long segments of AF ECGs [7]-[10].

However, some issues and open challenges can compromise or limit the performance of Hankel-based BTD in this particular application:

1) There is still no optimal automated method for atrial source selection.

2) The Hankel-based BTD was only assessed in segments with long R-R intervals and with well defined AA, visible in most part of the segment. However, short R$\mathrm{R}$ intervals $(<0.75 \mathrm{~s})$ and weak AA (amplitude of the 
f waves lower than $0.1 \mathrm{mV}$ ) are quite more common during persistent AF episodes.

Aiming to provide a way to alliviate these issues and make the tensor approach applicable in a wider range of situations, this paper proposes another approach to estimate the AA in challenging cases of AF, where the matrix-based techniques and even the Hankel-based BTD cannot provide a satisfactory performance. The BTD built from Löwner matrices proposed as a solution for BSS of rational functions in [11] is applied in this work, for the first time, to separate the VA from AA in AF ECGs. This method suits the characteristics of VA in an ECG recording, since the QRS complex can be well approximated by rational functions [12], [13], and when mapped onto Löwner matrices, the degree of the rational function matches with the rank of the Löwner matrix [11]. The VA estimated by the Löwner-based BTD is then subtracted from the ECG signal, resulting in a signal that mainly contains AA. Through this approach, no method for atrial source selection is needed, since the resulting signal is already the desired signal. Since the VA is a less complex signal than AA in AF, the multilinear rank of this component is lower and varies in a shorter range, which makes its estimation simpler.

Furthermore, the present work assesses BTD for the first time in segments with short R-R intervals and with disorganized (f waves with no sawtooth pattern) and/or weak AA of a population of patients with persistent $\mathrm{AF}$, comparing it to two popular matrix-based methods for AA extraction: RobustICAf [14] and PCA, as well as the Hankel-based BTD. Also, the estimation of the number of blocks based on the singular value decomposition (SVD) and the use of only 8 independent ECG leads are presented for the first time in a tensor approach for AA analysis in AF episodes.

\section{Block Term Decomposition AND ECG DATA}

The BTD of an arbitrary third-order tensor $\mathcal{T} \in \mathbb{R}^{I_{1} \times I_{2} \times I_{3}}$ is written as:

$$
\mathcal{T}=\sum_{r=1}^{R} \mathbf{E}_{r} \circ \mathbf{c}_{r}
$$

where $\circ$ represents the outer product, $\mathbf{c}_{r} \in \mathbb{R}^{I_{3}}$ is nonzero and $\mathbf{E}_{r} \in \mathbb{R}^{I_{1} \times I_{2}}$ has rank $L_{r}$, admitting the factorization $\mathbf{E}_{r}=\mathbf{A}_{r} \mathbf{B}_{r}^{T}$, where $\mathbf{A}_{r} \in \mathbb{R}^{I_{1} \times L_{r}}$ and $\mathbf{B}_{r} \in \mathbb{R}^{I_{2} \times L_{r}}$ have rank $L_{r}$. We may then rewrite (1) as:

$$
\mathcal{T}=\sum_{r=1}^{R}\left(\mathbf{A}_{r} \mathbf{B}_{r}^{T}\right) \circ \mathbf{c}_{r} .
$$

One can see that the BTD is a decomposition of $\mathcal{T}$ in multilinear rank- $\left(L_{r}, L_{r}, 1\right)$ terms, represented by a sum of the outer product of its matrix and vector factors, as shown in Figure 1. Several conditions guarantee the essential uniqueness of this tensor decomposition. For example, in [15, Theorem 2.2], it is shown that the BTD is essentially unique if the following conditions are satisfied:

1) The matrix factors $\mathbf{A}=\left[\begin{array}{llll}\mathbf{A}_{1} & \mathbf{A}_{2} & \ldots & \mathbf{A}_{R}\end{array}\right] \in$ $\mathbb{R}^{I_{1} \times \sum_{r=1}^{R} L_{r}}$ and $\mathbf{B}=\left[\begin{array}{llll}\mathbf{B}_{1} & \mathbf{B}_{2} & \ldots & \mathbf{B}_{R}\end{array}\right] \in$

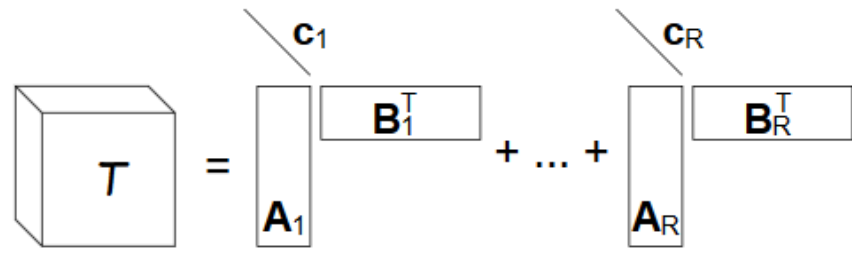

Fig. 1: Visual representation of BTD of an arbitrary third-order tensor.

$\mathbb{R}^{I_{2} \times \sum_{r=1}^{R} L_{r}}$ are full-column rank, which requires in particular that, $\sum_{r=1}^{R} L_{r} \leq I_{1}, I_{2}$.

2) Matrix $\mathbf{C}=\left[\begin{array}{llll}\mathbf{c}_{1} & \mathbf{c}_{2} & \ldots & \mathbf{c}_{R}\end{array}\right] \in \mathbb{R}^{I_{3} \times R}$ does not contain proportional columns.

Essentially unique means that the $r_{1}^{\text {th }}$ and $r_{2}^{\text {th }}$ terms can only be permuted when they have equal ranks and when $\mathbf{E}_{r}$ can only be scaled if $\mathbf{c}_{r}$ is counterscaled [11]. Milder uniqueness conditions can be found in [15].

Now, ECG recordings from $K$ leads composed by $N$ time samples can be viewed as a matrix:

$$
\mathbf{Y}=\mathbf{M S} \in \mathbb{R}^{K \times N}
$$

where $\mathbf{M} \in \mathbb{R}^{K \times R}$ is the mixing matrix, modeling the propagation of the cardiac electrical sources from the heart to the body surface, $\mathbf{S} \in \mathbb{R}^{R \times N}$ is the source matrix that contains mainly the atrial and ventricular sources, and $R$ is the number of sources.

BTD is proposed as a technique to solve BSS problems like (3) in [15] and its Löwner construction was detailed and exploited in [11]. The idea to tensorize the data $\mathbf{Y}$ is to map each of its rows onto a Löwner matrix $\mathbf{L}^{(k)} \in \mathbb{R}^{I \times J}, k=$ $1,2, \ldots, K$, whose $(i, j)$-entry is given by:

$$
L_{i, j}^{(k)}=\frac{y^{(k)}\left(x_{i}\right)-y^{(k)}\left(z_{j}\right)}{x_{i}-z_{j}},
$$

where $I+J=N$ and $y^{(k)}$ is the $k^{\text {th }}$ lead of the ECG data matrix sampled in a point set $T=\left\{t_{1}, t_{2}, \ldots, t_{N}\right\}$, which is partitioned in two different point sets: $X=\left\{x_{1}, x_{2}, \ldots, x_{I}\right\}$ and $Z=\left\{z_{1}, z_{2}, \ldots, z_{J}\right\}$. In this work, we consider signals with even samples and an interleaved partitioning, i.e., $I=$ $J=N / 2, X=\left\{t_{1}, t_{3}, \ldots, t_{N-1}\right\}$, and $Z=\left\{t_{2}, t_{4}, \ldots, t_{N}\right\}$, already used with success in ECG processing [11], although in a different problem.

Next, the tensor is built by stacking each Löwner matrix along the third dimension (as frontal slices) of a third-order tensor $\mathcal{Y} \in \mathbb{R}^{I \times J \times K}$, that is:

$$
\mathbf{Y}_{. . k}=\mathbf{L}^{(k)}=\sum_{r=1}^{R} m_{k, r} \mathbf{L}_{\mathbf{S}}^{(r)},
$$

where $\mathbf{L}_{\mathbf{S}}^{(r)} \in \mathbb{R}^{I \times J}$ is a Löwner matrix built from the $r^{\text {th }}$ row of $\mathbf{S}$. One can see that for each $r$, the outer product between matrix $\mathbf{L}_{\mathbf{S}}^{(r)}$ and the $r^{\text {th }}$ column of $\mathbf{M}$, i.e., $\mathbf{m}_{r}$, is being performed to build a third-order tensor containing the contribution of the $r^{\text {th }}$ source to the observed ECG. Putting 
together the contribution of all sources, the third-order tensor $\mathcal{Y}$ can be written as:

$$
\mathcal{Y}=\sum_{r=1}^{R} \mathbf{L}_{\mathbf{S}}^{(r)} \circ \mathbf{m}_{. r} .
$$

Comparing Equations (1) and (6), it can be seen that the ECG data tensor follows a BTD tensor model with the following correspondence:

$$
\left(\mathcal{T}, \mathbf{E}_{r}, \mathbf{c}_{r}, I_{1}, I_{2}, I_{3}, R\right) \Longleftrightarrow\left(\mathcal{Y}, \mathbf{L}_{\mathbf{S}}^{(r)}, \mathbf{m}_{. r}, I, J, K, R\right) .
$$

In the ECG, QRS complexes can be approximated by rational functions of low degree [11]-[13]:

$$
s(t)=a(t)+\sum_{f=1}^{F} \sum_{d=1}^{D f} \frac{c_{f, d}}{\left(t-p_{f}\right)^{d}},
$$

where $a(t)$ is a polynomial of degree $A, p_{f}$ are the complex poles, $t$ is the discrete time, and $c_{f, d}=1 /\left(u_{f}-v_{d}\right)$ are the scalar entries of a Cauchy matrix based on the vectors $\mathbf{u} \in \mathbb{C}^{I}$ and $\mathbf{v} \in \mathbb{C}^{J}$, with $u_{i} \neq v_{j}, \forall i, j$. This way, their associated Löwner matrices accept the general decomposition [11]:

$$
\mathbf{L}=\mathbf{Z G} \tilde{\mathbf{Z}}^{T}
$$

where $\mathbf{Z} \in \mathbb{C}^{I \times L}$ and $\tilde{\mathbf{Z}} \in \mathbb{C}^{J \times L}$ have $\operatorname{rank} L=A+$ $\sum_{f=1}^{F} D_{f}$, and $\mathbf{G} \in \mathbb{C}^{L \times L}$ is a block-diagonal matrix with Hankel and upper antitriangular matrices $\mathbf{G}_{W}$ and $\mathbf{G}_{f, D_{f}}$ for $1 \leq f \leq F$ along its diagonal.

The cases where $s(t)$ has coinciding and non-coinciding poles, and is a polynomial of degree $W$ are detailed in [11], as well as general uniqueness conditions of (6).

\section{Measuring Atrial Activity Content}

The measurement of the estimation quality (or the AA content) of real signals is a difficult task. Since there is no ground truth for comparison, one needs to take advantage of some features present in AA during AF episodes. For example, in the frequency domain, the AA during AF has a peak between 3 and $9 \mathrm{~Hz}$. The position of this peak is called dominant frequency (DF). In this section, two parameters used to measure AA extraction quality are presented. The first one is the spectral concentration (SC), defined as the relative amount of energy around the DF. The SC is computed as in [6]:

$$
S C=\frac{\sum_{f_{i}=0.82 f_{p}}^{1.17 f_{p}} P_{S}\left(f_{i}\right)}{\sum_{f_{i}=0}^{F_{s} / 2} P_{S}\left(f_{i}\right)}
$$

where $f_{p}$ is the value of the $\mathrm{DF}, F_{s}$ is the sampling frequency, $f_{i}$ is the discrete frequency and $P_{S}$ is the power spectrum of the source signal computed using Welch's method as in [6]. The second parameter, used to discard sources with irrelevant AA content, is the power contribution to lead V1, denoted $P(r)$, which is given by [9]:

$$
P(r)=\frac{1}{N}\left\|m_{V 1, r} \mathbf{s}_{r .}\right\|^{2}
$$
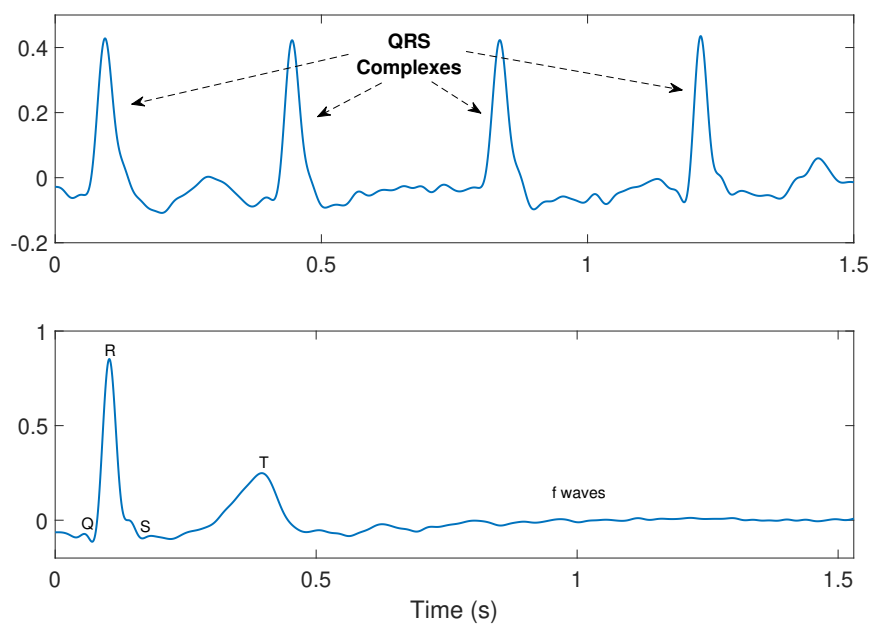

Fig. 2: Top: a 1.5-second segment of an AF ECG recording (in $\mathrm{mV}$ ) with $4 \mathrm{QRS}$ complexes from one patient (P2). The $\mathrm{R}-\mathrm{R}$ intervals are short and represented by the time difference between the peaks. Bottom: a single heartbeat segment of an AF ECG recording (in $\mathrm{mV}$ ) with a weak AA signal, from another observed patient (P6). Only bipolar limb lead II is shown for clarity, although the 8 independent leads (I, II, V1V6) are processed in the experiments reported here.

in $\mathrm{mV}^{2}$, where symbol $\|\cdot\|$ represents the $l_{2}$-norm, $m_{V 1, r}$ is the contribution of the $r^{\text {th }}$ source to lead V1 and $\mathbf{s}_{r}$. is the $r^{\text {th }}$ source in time domain. The $P(r)$ of an AA source is expected to be relatively strong $\left(>10^{-4} \mathrm{mV}^{2}\right)$, since this lead is the one that typically best reflects AA in AF ECGs.

\section{Database And Experimental Setup}

\section{A. Real AF ECG Data and Preprocessing}

All the recordings belong to a database provided by the Cardiology Department of Princess Grace Hospital Center, Monaco. The recordings are acquired at a $977 \mathrm{~Hz}$ sampling rate and are preprocessed by a zero-phase forward-backward type-II Chebyshev bandpass filter with cutoff frequencies of 0.5 and $40 \mathrm{~Hz}$, in order to suppress high-frequency noise and baseline wandering.

Experiments are performed in 10 different segments of ECG recordings from 10 different patients suffering from persistent AF, where 5 of these segments have a disorganized and/or weak AA, and the other 5 have very short R-R intervals. In order to exploit all spatial diversity, while reducing the computational cost, only 8 independent leads are processed (I, II, V1-V6). The two type of segments used in the reported experiments are shown in lead II in Figure 2.

The 5 segments with short R-R intervals from 5 different patients (P1-P5) have 1.5 seconds of duration with at least 2 QRS complexes. The other 5 segments with disorganized and/or weak AA, from other 5 different patients (P6-P10), are composed by one heartbeat, i.e., the QRS complex followed by the $\mathrm{T}$ wave and the visible $\mathrm{f}$ waves, and have between 
1.3-1.7 seconds. All the 10 segments are downsampled by a factor of 2 , since the originally built third-order tensors pose some difficulties to be processed by Tensorlab MATLAB toolbox [17].

\section{B. BTD Setup}

The BTD is implemented using the non-linear least squares (NLS) method available in Tensorlab MATLAB toolbox. For the Hankel-based BTD, the choice $R=6$ and $L_{r}=48$, for $r=1,2, \ldots, R$, is made. The choice of $R$ is based on the SVD of the observed data matrix, taking into account the most significant singular values. The choice of $L_{r}$ is based on the work of [8], that showed that this value provided good results for the heartbeat with the largest TQ segment of one of the patients considered in the present work. Also, the work of [10] showed that this value provides satisfactory results for consecutive segments of the whole ECG recording of the same patient.

For the Löwner-based BTD, the choice $R=\{2,3\}$ and $L_{r}=L$, for $r=1,2, \ldots, R$, with $L$ taking values in the set $\{3,4,5,6\}$ is made. This choice is made based on previous experiments that aimed at estimating only the VA from the original recording, and then subtract it from the ECG, providing the signal with mainly AA content. Previous experiments show that the VA subspace is mainly present in the first 2 or 3 principal singular values of the ECG matrix, with low rank values.

For both techniques, the maximum number of iterations is set to 1000. Monte Carlo runs with Gaussian random initialization for the spatial and temporal factors at each run are used to analyze the performance of BTD in each segment regarding the separation of VA from AA. Monte Carlo runs are needed since the performance of BTD depends strongly on the initialization of its factors and a suitable initialization is still an open challenge.

\section{EXPERIMENTAL RESULTS}

\section{A. Segments With Short R-R Intervals}

Table I shows the values of SC in \% of the estimated AA and the number of iterations until convergence of the NLS method for the Löwner and Hankel-based BTDs. The best performance of the ten independent runs is shown. Patients P1-P5 correspond to the patients whose segments have short $\mathrm{R}-\mathrm{R}$ intervals. We can see in Table I that the SC of the AA, resulting from the subtraction of VA from the ECG, for the Löwner-based BTD is always higher than the one estimated by the Hankel-based BTD. Moreover, the mean of the number of iterations of the NLS method until convergence is always shorter, meaning that the proposed approach provides a better performance with less computational cost. In addition, the Löwner-based BTD could separate the VA from AA in all the population of patients whose segments have a short R-R intervals, while the Hankel-based BTD could not successfully separate them for the patient P5. The DF of both tensor approaches lies in the interval $5.48-7.15 \mathrm{~Hz}$, while the $P(r)$ values of the Löwner-based BTD lie in the interval
TABLE I: SC values (\%) of the AA and the mean of the number of iterations of the NLS method $(\mu)$ with the standard deviation $(\sigma)$ for the Löwner-based BTD (BTD-L) and the Hankel-based BTD (BTD-H) of ten Monte Carlo runs.

\begin{tabular}{|c|c|c|c|c|}
\hline \multirow{2}{*}{ Patients } & \multicolumn{2}{|c|}{ BTD-L } & \multicolumn{2}{c|}{ BTD-H } \\
\cline { 2 - 5 } & SC (\%) & $\boldsymbol{\mu} \pm \boldsymbol{\sigma}$ & SC (\%) & $\boldsymbol{\mu} \pm \boldsymbol{\sigma}$ \\
\hline \hline P1 & 51.2 & $60 \pm 18.1$ & 44.1 & $185.7 \pm 47.2$ \\
\hline P2 & 54.6 & $60.3 \pm 22.4$ & 33.8 & $181 \pm 68.1$ \\
\hline P3 & 46.1 & $46.7 \pm 15.4$ & 30.9 & $164.9 \pm 58.1$ \\
\hline P4 & 63.5 & $125.3 \pm 36.5$ & 61.9 & $143.5 \pm 37.1$ \\
\hline P5 & 33.7 & $44 \pm 16.9$ & none & none \\
\hline \hline P6 & 60.1 & $28.2 \pm 6.7$ & none & none \\
\hline P7 & 60.0 & $29.9 \pm 11.5$ & none & none \\
\hline P8 & 52.9 & $33.3 \pm 18.2$ & none & none \\
\hline P9 & 40.7 & $35 \pm 12.8$ & none & none \\
\hline P10 & 56.9 & $36.8 \pm 12.1$ & none & none \\
\hline
\end{tabular}

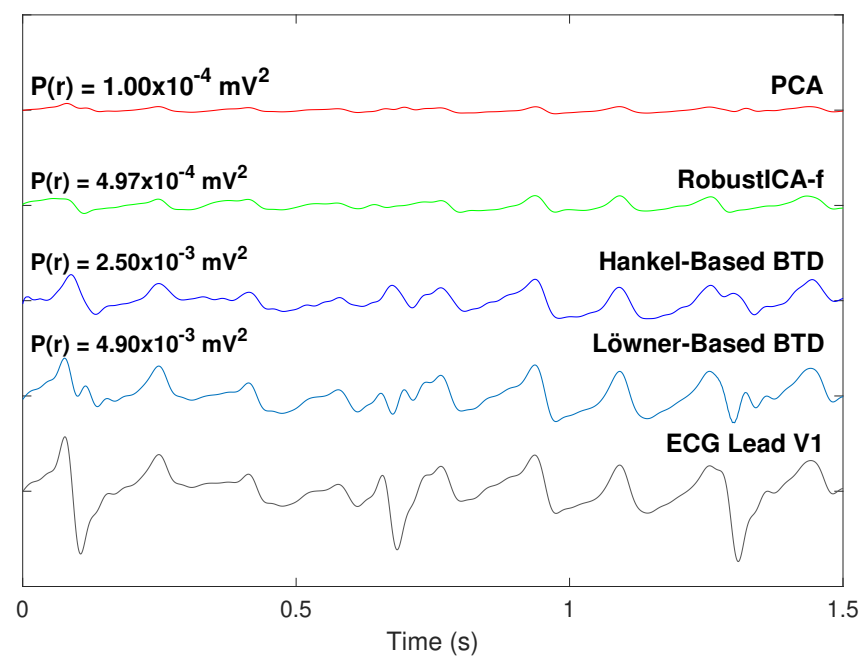

Fig. 3: Estimated atrial sources contribution to lead V1 in a segment of short R-R intervals of Patient P4 by Hankel and Löwner-based BTDs, RobustICA-f and PCA in the time domain. AA signal estimates are vertically shifted for clarity with their respectives power contributions to lead V1.

$7.2 \times 10^{-4}-9.1 \times 10^{-3} \mathrm{mV}^{2}$, which are consistently higher than the $P(r)$ values of the Hankel-based one, that lie in the interval $5.7 \times 10^{-4}-2.5 \times 10^{-3} \mathrm{mV}^{2}$.

Regarding the performance of the matrix-based techniques, PCA could only successfully separate VA from AA in patient $\mathrm{P} 4$, with a SC of $55.4 \%$ and DF $=5.72 \mathrm{~Hz}$, while RobustICA$\mathrm{f}$ could only separate them in patients $\mathrm{P} 1$ and $\mathrm{P} 4$, with SCs and DFs equal to $40.7 \% / 6.91 \mathrm{~Hz}$ and $60.8 \% / 5.72 \mathrm{~Hz}$, respectively.

In Figure 3 we can see the observed segment of Patient P4, the only patient where the 4 signal processing techniques could successfully separate the sources. The proposed technique provides a signal with clearer AA content and a higher $P(r)$ value than the other methods. 


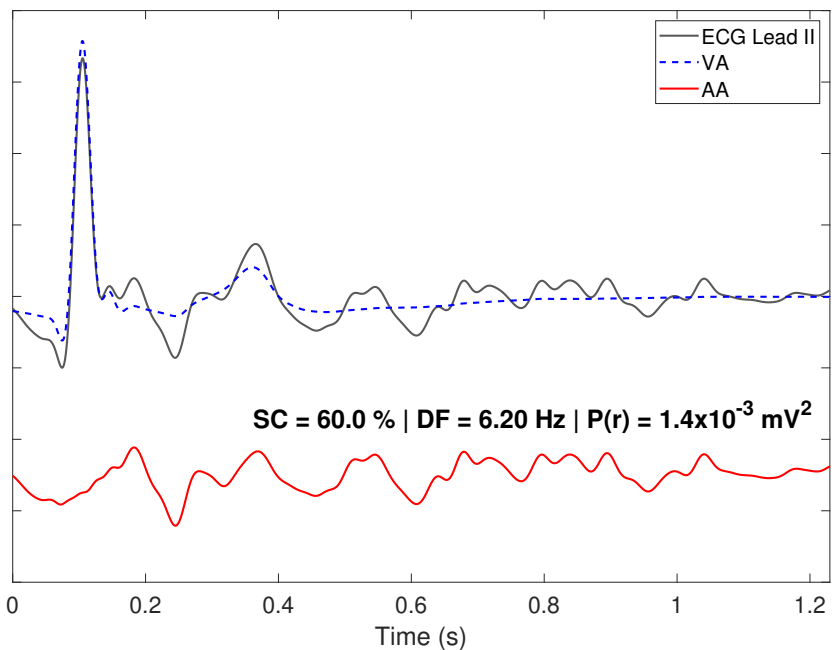

Fig. 4: Original recording, VA and AA estimates in lead II by the Löwner-based BTD of Patient P7. The AA signal was vertically shifted for clarity.

\section{B. Segments With Disorganized and/or Weak AA}

For the patients whose segments are characterized by a very weak and/or disorganized AA contribution, the matrix-based methods and the Hankel-based BTD could not clearly separate the sources. The AA signal was not identified due to its very weak content or, in some cases, significant VA residuals in the signal. For the Löwner-based BTD, the AA signal was successfully separated from the VA with a relatively satisfactory performance, considering the nature of the recording.

In Table I, patients P6-P10 correspond to the patients whose segments have disorganized and/or weak AA contribution. We can see that a relatively low number of iterations provide a satisfactory performance regarding the quality of AA content. The values of $P(r)$ lie in the range of $4.6 \times 10^{-4}-1.7 \times 10^{-3}$ $\mathrm{mV}^{2}$, showing that each source have significant AA content.

In Figure 4 we can see the observed recording, as well as the estimated VA and AA of the processed segment of one of the patients in the observed population, for the best performance of ten Monte Carlo runs. The segments are shown in lead II for a better clarity of the estimated VA. It can be seen that even the $\mathrm{T}$ wave was estimated by the proposed method, that is, not only the ventricular depolarization, but also its repolarization were estimated.

\section{CONCLUSION}

The present work has proposed a new tensor approach to separate VA and AA in persistent AF ECG recordings. This tensor method is the BTD built from Löwner matrices, used to model and estimate only the VA and then subtract it from the original recording, resulting in the desired AA. The BTD approach was applied for the first time in AF segments with short R-R intervals, which are quite common in persistent $\mathrm{AF}$, and with a disorganized and/or weak AA contribution, also common in this stage of the arrhythmia. Experiments based on Monte Carlo simulations have shown the improved performance of the Löwner-based BTD over the one built from Hankel matrices and the well-known matrix-based techniques RobustICA-f and PCA, regarding AA extraction quality in this challenging clinical setting.

A more detailed theoretical and experimental analysis of this new tensor approach in AF ECGs should be presented in following works. In particular, experiments should be performed in a larger population of patients in order to provide more relevant clinical results.

\section{REFERENCES}

[1] C. T. January, L. S. Wann, J. S. Alpert, H. Calkins, J. C. Cleveland, J. E. Cigarroa, J. B. Conti, et al., "2014 AHA/ACC/HRS guideline for the management of patients with atrial fibrillation: A report of the American College of Cardiology/American Heart Association Task Force on practice guidelines and the Heart Rhythm Society", Circulation, vol. 64, no. 21, pp. 2246-2280, Dec. 2014.

[2] L. Mainardi, L. Sörnmo, S. Cerutti, "Understanding Atrial Fibrillation: The Signal Processing Contribution", Synthesis Lectures on Biomedical Engineering, Morgan \& Claypool Publishers, 2008.

[3] J. J. Rieta, F. Castells, C. Sánchez, V. Zarzoso, and J. Millet, "Atrial activity extraction for atrial fibrillation analysis using blind source separation", IEEE Transactions on Biomedical Engineering, vol. 51, no. 7, pp. 1176-1186, Jul. 2004.

[4] I.T. Jolliffe, Principal Component Analysis, Wiley Online Library, 2005.

[5] P. Comon, "Independent component analysis, A new concept?", Signal Processing, no. 36, pp. 287-314, 1994.

[6] F. Castells, J. J. Rieta, J. Millet, and V. Zarzoso, "Spatiotemporal blind source separation approach to atrial activity estimation in atrial tachyarrhythmias", IEEE Transactions on Biomedical Engineering, vol. 52, no. 2, pp. 258-267, Feb. 2005.

[7] L. N. Ribeiro, A. R. Hidalgo-Muñoz, and V. Zarzoso, "Atrial signal extraction in atrial fibrillation electrocardiograms using a tensor decomposition approach", in Proc. EMBC-2015, 37th Annual International Conference of the IEEE Engineering in Medicine and Biology Society, Milan, Italy, Aug. 25-29, pp. 6987-6990, 2015.

[8] V. Zarzoso, "Parameter estimation in block term decomposition for noninvasive atrial fibrillation analysis", in Proc. CAMSAP-2017, IEEE International Workshop on Computational Advances in Multi-Sensor Adaptive Processing, Curaçao, Dutch Antilles, Dec. 10-13, 2017.

[9] P. M. R. de Oliveira and V. Zarzoso, "Source analysis and selection using block term decomposition in atrial fibrillation", in Proc. LVA/ICA-2018, 14th International Conference on Latent Variable Analysis and Signal Separation, Guildford, United Kingdom, Jul. 2-6, pp. 46-56, 2018.

[10] P. M. R. de Oliveira and V. Zarzoso, "Temporal Stability of Block Term Decomposition in Noninvasive Atrial Fibrillation Analysis", in Proc. Asilomar-2018, 52nd Annual Asilomar Conference on Signals, Systems, and Computers, Pacific Grove, U.S.A, Oct. 28-31, pp. 816-820, 2018.

[11] O. Debals, M. V. Barel, and L. De Lathauwer, "Löwner-based blind signal separation of rational functions with applications", IEEE Transactions on Signal Processing, vol. 64, no. 8, pp. 1909-1918, 2016.

[12] L. Lócsi, "Approximating poles of complex rational functions", Acta Universitatis Sapientiae, Mathematica, vol. 1, pp. 169-182, 2009.

[13] S. Fridli, L. Lócsi, and F. Schipp, "Rational functions systems in ECG processing", in Proc. EUROCAST-2011, Part I, pp. 88-95, 2012.

[14] V. Zarzoso and P. Comon, "Robust independent component analysis by iterative maximization of the kurtosis contrast with algebraic optimal step size", IEEE Transactions on Neural Networks, vol. 21, no. 2, pp. 248-261, 2010.

[15] L. De Lathauwer, "Blind separation of exponential polynomials and the decomposition of a tensor in rank- $\left(l_{r}, l_{r}, 1\right)$ terms", SIAM Journal on Matrix Analysis and Applications, vol. 32, no. 4, pp. 1451-1474, 2011.

[16] D. L. Boley, F. T. Luk, and D. Vandevoorde, "Vandermonde factorization of a Hankel matrix", in Proceedings of the Workshop on Scientific Computing, Hong Kong, March 1997.

[17] N. Vervliet, O. Debals, L. Sorber, M. Van Barel, and L. De Lathauwer, Tensorlab 3.0, Available online, Mar. 2016. URL: https://www.tensorlab.net/ 